manuscripta mathematica manuscript No.

(will be inserted by the editor)

Hanspeter Kraft · Lance W. Small · Nolan R. Wallach

\title{
Properties and Examples of FCR-Algebras
}

Received: date / Revised version: date

\begin{abstract}
An algebra $A$ over a field $k$ is FCR if every finite dimensional representation of $A$ is completely reducible and the intersection of the kernels of these representations is zero. We give a useful characterization of FCR-algebras and apply this to $C^{*}$-algebras and to localizations. Moreover, we show that "small" products and sums of FCR-algebras are again FCR.
\end{abstract}

\section{Introduction}

It is well-known that the enveloping algebra $\mathfrak{U}(\mathfrak{g})$ of a semisimple Lie algebra $\mathfrak{g}$ in characteristic zero has the following remarkable properties:

- Every finite dimensional representation of $\mathfrak{U}(\mathfrak{g})$ is completely reducible.

- $\mathfrak{U}(\mathfrak{g})$ is residually finite dimensional, i.e., the intersection of the kernels of all finite dimensional representations is zero.

The first property is due to WeYL, the second to Harish-Chandra (see [Dix77], 2.5.7, p. 84).

Question. Are there other algebras satisfying these properties and how do they look?

We introduced the following notion in [KrS94] in order to study this question.

Definition. An algebra $A$ over an arbitrary field $k$ is an FCR-algebra if every finite dimensional representation of $A$ is completely reducible and $A$ is residually finite-dimensional.

$(\mathrm{FCR}=$ "Finite dimensional representations are Completely Reducible")

At that time the only known examples of FCR-algebras beside the finite dimensional semisimple algebras were the enveloping algebras of semisimple

H. Kraft: Mathematisches Institut, Universität Basel, Rheinsprung 21, CH-4051 Basel, Switzerland (e-mail: kraft@math.unibas.ch)

L.W. Small and N.R. Wallach: Department of Mathematics, University of California, San Diego, 9500 Gilman Drive, La Jolla, CA 92093-0112, USA (e-mail: lwsmall@ucsd.edu, nwallach@ucsd.edu) 
Lie algebras (in characteristic zero) and the quantum enveloping algebras $\mathfrak{U}_{q}(\mathfrak{g})$ for $q$ not a root of unity. Since then a number of new examples of FCRalgebras have been discovered: Invariant algebras of enveloping algebras and direct summands ([KrS94], [KSW99]), algebras of invariant differential operators ([MvB98], [Sch00]), generalized Weyl algebras and orthosymplectic Lie superalgebras ([KiS00]).

The aim of this paper is to give a simple, but useful characterization of FCR-algebras (section 3) and to apply this to $C^{*}$-algebras (section 3 ) and to localizations (section 4). Moreover, we show that "small" direct products and direct sums (with a unit adjoined) of FCR-algebras are again FCR (section 2).

Remark 1. The classification of (affine Noetherian) FCR-algebras is an open problem, even in small dimensions. It is not hard to see that there are no such algebras in GELFAND-KIRILLOW-dimension (GK-dimension) equal to 1. (In fact, it follows from [SSW85] that any such algebra is PI and from [Fa87] that a (finitely generated) FCR-PI-algebra is finite dimensional.)

The smallest examples known so far are $\mathfrak{U}\left(\mathfrak{s l}_{2}\right)$ and its invariant subalgebras under a finite group of automorphism, all in GK-dimension 3 (see [KrS94]). We conjecture that there are no FCR-algebras in GK-dimension 2. At this time, we can only show that any residually finite homomorphic image of an enveloping algebra has GK-dimension $\geq 3$.

\section{Products and sums}

Theorem 1. Let $\left(A_{i}\right)_{i \in I}$ be a family of FCR-algebras over a field $k$. Assume that the cardinality of $k$ is greater or equal than the cardinality of the index set $I$. Then the product $\prod_{i \in I} A_{i}$ is an FCR-algebra.

Theorem 2. Let $\left(A_{i}\right)_{i \in I}$ be a family of FCR-algebras over a field $k$. Then the sum $k \oplus \bigoplus_{i \in I} A_{i}$ is an FCR-algebra.

(We add a copy of $k$ and define multiplication by $(\lambda, a) \cdot(\mu, b)=(\lambda \mu, \lambda b+$ $\mu a+a b)$ so that we have again a $k$-algebra with a unit. Clearly, $k \oplus \bigoplus_{i \in I} A_{i} \subset$ $\prod_{i \in I} A_{i}$ is a (unital) subalgebra in a natural way.)

It is clear that both algebras are residually finite dimensional, since the intersection of the kernels of the projection homomorphisms

$$
\operatorname{pr}_{j}: A:=\prod_{i \in I} A_{i} \rightarrow A_{j}
$$

and of the homomorphisms

$p_{j}: A:=k \oplus \bigoplus_{i \in I} A_{i} \rightarrow A_{j},(\lambda, a) \mapsto \lambda+a_{j} \quad$ and $\quad \operatorname{pr}: A:=k \oplus \bigoplus_{i \in I} A_{i} \rightarrow k$

is zero in both cases. So the main point is to prove that every finite dimensional representation is completely reducible. We will do this by showing 
that for every surjective $k$-algebra homomorphism $A \rightarrow B$ where $B$ is a finite dimensional algebra, there is a finite subproduct $\prod_{\text {finite }} A_{i_{\nu}} \subset A$ which maps surjectively onto $B$. This reduces the proof to the following result.

Proposition 1. A finite product of FCR-algebras is FCR.

Proof. This is clear: Every homomorphism $\prod_{i=1}^{n} A_{i} \rightarrow B$ onto a finite dimensional algebra $B$ factors through a product $\prod_{i=1}^{n} A_{i} / \mathfrak{a}_{i}$ where the algebras $A_{i} / \mathfrak{a}_{i}$ are finite dimensional and hence semisimple.

Proof of Theorem 2. Let $\varphi: A:=k \oplus \bigoplus_{i \in I} A_{i} \rightarrow B$ be a homomorphism onto a finite dimensional algebra $B$. Lifting a $k$-basis of $B$ to $A$ we see that there is a finite sum $k \oplus \bigoplus_{\text {finite }} A_{i_{\nu}}$ which contains all these representatives. Therefore, $\varphi\left(k \oplus \bigoplus_{\text {finite }} A_{i_{\nu}}\right)=B$. As a $k$-algebra, the sum $k \oplus \bigoplus_{\text {finite }} A_{i_{\nu}}$ is isomorphic to $k \times \prod_{\text {finite }} A_{i_{\nu}}$, hence is FCR by Proposition 1 .

For the proof of the first theorem we need some preparation.

Lemma 1. Let $k$ be a field and consider the product $C:=\prod_{I} k$ where the index set $I$ has a cardinality less or equal to the cardinality of $k$. Then every $k$-homomorphism $C \rightarrow B$ into a finite dimensional $k$-algebra $B$ factors through a projection onto a finite product $\prod_{I} k \rightarrow \prod_{\text {finite }} k$. In particular, the image of $C$ in $B$ is a finite product $k \times k \times \cdots \times k$.

Proof. For any subset $J \subset I$ we denote by $e_{J}$ the idempotent defined by

$$
\left(e_{J}\right)_{i}= \begin{cases}1 & \text { if } i \in J, \\ 0 & \text { if } i \notin J .\end{cases}
$$

We have to show that the kernel of any $k$-homomorphism $\varphi: \prod_{I} k \rightarrow B$, $B$ finite dimensional, contains an idempotent $e_{J}$ where $J \subset I$ is cofinite. In fact, this means that the kernel contains $C e_{J}=\prod_{j \in J} k$, hence the homomorphism factors through $C / C e_{J}=\prod_{i \in I \backslash J} k$.

Because of the assumption we can choose an element $a \in C$ such that all components $a_{i}$ are distinct. The image $b:=\varphi(a) \in B$ satisfies an equation $\sum_{s=0}^{n} \lambda_{s} b^{s}=0$ where $\lambda_{s} \in k$ and $\lambda_{n}=1$. The polynomial $p(x):=\sum_{s=0}^{n} \lambda_{s} x^{s}$ has only finitely many zeros in $k$. Therefore, $p\left(a_{i}\right)=0$ for only finitely many $i \in I$. This implies that the support $J$ of the element $p(a) \in \operatorname{ker} \varphi$ is cofinite. (The support of an element $c \in C$ is defined to be the subset $\left\{i \in I \mid c_{i} \neq 0\right\}$.) Therefore, the kernel contains $C p(a)=C e_{J}$ and we are done.

Proof of Theorem 1. Let $\varphi: \prod_{i \in I} A_{i} \rightarrow B$ be a surjective homomorphism onto a finite dimensional $k$-algebra $B$. We have $\prod_{I} k \subset \prod_{i \in I} A_{i}$ in a natural way. By our Lemma 1 above there is an idempotent $e_{J} \in \operatorname{ker} \varphi \cap \prod_{I} k$ with cofinite $J \subset I$. Hence, the homomorphism $\varphi$ factors through a finite product

$$
\varphi: \prod_{i \in I} A_{i} \rightarrow \prod_{\text {finite }} A_{i_{\nu}} \rightarrow B
$$

and we are done by Proposition 1. 
Remark 2. We do not know if Theorem 1 holds without the assumption on the cardinality of the field $k$. However, our argument breaks down as shown by the following example. Consider the product $A:=\prod_{\mathbb{N}} \mathbb{F}_{2}$. The maximal ideals in this algebra correspond bijectively to the ultrafilters of $\mathbb{N}$. It is well-known that there are many ultrafilters which are different from the principal filters (i.e. those filters consisting of all subsets containing a given element $i \in \mathbb{N}$, corresponding to the maximal ideal $\prod_{\mathbb{N} \backslash\{i\}} \mathbb{F}_{2}$.). However, we have $A / M=\mathbb{F}_{2}$ for every maximal ideal $M$ of $A$.

On the other hand we will see later that every product of matrix rings over division rings is FCR (section 3, Corollary 1).

\section{A characterization of FCR-algebras}

Theorem 3. Let $A$ be a $k$-algebra where $k$ is an arbitrary field. The following assertions are equivalent:

(i) Every finite dimensional representation of $A$ is completely reducible.

(ii) For every two-sided ideal $I \subset A$ of finite codimension we have $I^{2}=I$.

(iii) If $M_{1}, M_{2}$ are two-sided maximal ideals of finite codimension then we have $M_{1} \cap M_{2}=M_{1} M_{2}=M_{2} M_{1}$.

(The last statement includes the condition $M^{2}=M$ for every two-sided maximal ideal of finite codimension.)

Proof. (i) $\Rightarrow$ (ii): Let $I \subset A$ be a two-sided ideal of finite codimension. Then $A / I$ is a finite dimensional semisimple algebra and $I / I^{2}$ is a semisimple $A / I$-module. If $I$ where different from $I^{2}$ then we can find an $A$-submodule $K / I^{2} \subset I / I^{2}$ of finite codimension such that the quotient $I /\left(K+I^{2}\right)$ is nontrivial. If follows that $M:=A /\left(I^{2}+K\right)$ is finite dimensional. Therefore, we have an exact sequence

$$
0 \rightarrow I / K \rightarrow M \rightarrow A / I \rightarrow 0
$$

of finite dimensional $A$-modules. By assumption, these modules are semisimple and thus the sequence splits. Hence $M$ is annihilated by $I$, and so $I /\left(I^{2}+K\right)=I M=0$, in contradiction to the choice of $K$. Thus we have $I=I^{2}$.

(ii) $\Rightarrow$ (iii): Put $I:=M_{1} \cap M_{2}$. Then $I$ has finite codimension and we obtain $I^{2} \subset M_{1} M_{2} \cap M_{2} M_{1} \subset I$. Since $I^{2}=I$ by assumption, the claim follows.

(iii) $\Rightarrow$ (i): Let $J \subset A$ be a two-sided ideal of finite codimension. We have to show that the algebra $A / J$ is semisimple. Since $A / J$ is finite dimensional there are only finitely many two-sided maximal ideals $M_{1}, M_{2}, \ldots, M_{s}$ containing $J$, and

$$
\left(M_{1} \cap M_{2} \cap \ldots \cap M_{s}\right) / J \subset A / J
$$


is the nilradical of $A / J$. From the assumption we obtain, by an easy induction, that

$$
D:=M_{1} \cap M_{2} \cap \ldots \cap M_{s}=M_{\sigma(1)} M_{\sigma(2)} \cdots M_{\sigma(s)}
$$

for any permutation $\sigma$ of $\{1,2, \ldots, s\}$. Therefore, $D^{2}=D$ and so the nilradical $D / I$ is trivial.

We will now give an interpretation of statement (iii) in the Theorem above. Let us first look at an example.

Example 1. Consider the $k$-algebra $B:=\left[\begin{array}{ll}k & k \\ 0 & k\end{array}\right]$ and the two two-sided maximal ideals $M_{1}:=\left[\begin{array}{ll}k & k \\ 0 & 0\end{array}\right]$ and $M_{2}:=\left[\begin{array}{ll}0 & k \\ 0 & k\end{array}\right]$. We find

$$
M_{1} M_{2}=\left[\begin{array}{ll}
0 & k \\
0 & 0
\end{array}\right]=M_{1} \cap M_{2} \quad \text { and } \quad M_{2} M_{1}=(0)
$$

On the other hand, there is an exact non-split sequence of (left) $A$-modules

$$
0 \rightarrow A / M_{2} \rightarrow\left[\begin{array}{l}
k \\
k
\end{array}\right] \rightarrow A / M_{1} \rightarrow 0
$$

whereas $\operatorname{Ext}_{A}^{1}\left(A / M_{2}, A / M_{1}\right)=0$. This is a general fact as seen from the following lemma.

Lemma 2. Let $A$ be a k-algebra and $P_{1}, P_{2}$ two simple $A$-modules. Put $M_{i}:=\operatorname{Ann}_{A} P_{i}$. Then we have

$$
\operatorname{Ext}_{A}^{1}\left(P_{2}, P_{1}\right)=0 \quad \Longleftrightarrow \quad M_{1} M_{2}=M_{1} \cap M_{2} .
$$

Outline of Proof. Given any exact sequence $0 \rightarrow P_{1} \rightarrow P \rightarrow P_{2} \rightarrow 0$ of $A$ modules we see that $P$ is annihilated by the product $M_{1} M_{2}$. So if $M_{1} M_{2}=$ $M_{1} \cap M_{2}$ we obtain a surjective homomorphism $A / M_{1} \cap M_{2}=A / M_{1} \oplus$ $A / M_{2} \rightarrow P$ which implies that $P$ is semisimple.

On the other hand, every module $P$ annihilated by $M_{1} M_{2}$ admits an exact sequence $0 \rightarrow M_{2} P \rightarrow P \rightarrow P / M_{2} P \rightarrow 0$. Hence $P$ is an extension between an $A / M_{2}$-module $P / M_{2} P$ and an $A / M_{1}$-module $M_{2} P$. So if every such sequence splits, it follows that $P$ is annihilated by $M_{1} \cap M_{2}$ and so $M_{1} M_{2}=M_{1} \cap M_{2}$.

As an application of Theorem 3 we will show that all finite dimensional representations of a $C^{*}$-algebra are completely reducible.

Proposition 2. Let $A$ be a $C^{*}$-algebra with unit. Then every two-sided ideal I of finite codimension is closed, $*$-invariant and satisfies $I^{2}=I$. In particular, every finite dimensional representation of $A$ is completely reducible. 
Proof. (a) We start with a general remark about ideals in $C^{*}$-algebras. If $I \subset A$ is a closed two-sided ideal then $I$ is automatically $*$-invariant by Proposition 1.8 .2 of [Dix64]. It follows that $I$ is generated by hermitian elements, i.e. those $x \in I$ which satisfy $x^{*}=x$. Moreover, every hermitian $x \in I$ can be written in the form $x=x^{+}-x^{-}$where both $x^{+}$and $x^{-}$have a positive spectrum $([\operatorname{Dix} 64, ?])$ and belong to $I$. (We regard $I$ itself as a $C^{*}$-algebra.) Now we can use Proposition 1.6.1 of [Dix64] and conclude that $x=a^{2}-b^{2}$ where $a, b \in I$. (In particular, we have $I^{2}=I$.)

(b) Let $M$ be a two-sided maximal ideal of $A$. Then $M$ is closed: It's closure is again a two-sided ideal and cannot contain the unit element 1 of $A$, because every element of the form $1+x$ is invertible in $A$ for $\|x\|<1$.

Now let $M_{1}, M_{2}$ be two two-sided maximal ideals in $A$. Then $M_{1} \cap M_{2}$ is closed and therefore generated by elements of the form $x=a^{2}-b^{2}$ where $a, b \in M_{1} \cap M_{2}$ by (a). But this implies that $M_{1} \cap M_{2} \subset M_{1} M_{2}$. Thus statement (iii) of Theorem 3 is satisfied and the claim follows.

Let us recall here that a vON NEUMANN regular algebra $A$ is defined to be a $k$-algebra which satisfies the following condition: For every element $a \in A$ there is an $x \in A$ such that $a=$ axa (see [La91] Theorem 4.23). This implies that $I=I^{2}$ for every (left or right) ideal. Thus we obtain the following results.

Proposition 3. If $A$ is a VON NEUMANN regular algebra then every finite dimensional representation of $A$ is completely reducible.

Corollary 1. An arbitrary product $A=\prod_{i \in I} A_{i}$ of VON NEUMANN regular algebras $A_{i}$ is again VON NEUMANN regular and so every finite dimensional representation of $A$ is completely reducible. In particular, every product $\prod_{i \in I} M_{n_{i}}\left(K_{i}\right)$ of matrix rings over division rings $K_{i}$ is FCR.

(Cf. section 2, Remark 2)

\section{Localization}

We add some results about the localization of an FCR-algebra $R$ with respect to a (right) Ore set $S \subset R$. (For definitions and basic properties of non-commutative localization we refer to [La98, Chap. 4]. Since we do not exclude 0-divisors in $S$ we always assume that $S$ satisfies the following additional condition: If $s r=0$ for some $s \in S$ and $r \in R$ then there is an $s^{\prime} \in S$ such that $r s^{\prime}=0$.)

Proposition 4. Let $R$ be an FCR-algebra, $S \subset R$ be a right Ore set and $R_{S}$ the corresponding localization.

(1) Every finite-dimensional representation of $R_{S}$ is completely reducible.

(2) If $J \subset R_{S}$ is a two-sided ideal of finite codimension and $I:=J \cap R$ then $J=I R_{S}$ and $R / I \stackrel{\simeq}{\longrightarrow} R_{S} / J$. 
(3) If $S$ is generated by finitely many normalizing elements (i.e. elements $s$ satisfying $s R=R s$ ), then $R_{S}$ is $\mathrm{FCR}$. In this case, the two-sided maximal ideals of finite codimension of $R_{S}$ are of the form $M^{\prime}=M R_{S}=$ $R_{S} M$ where $M$ is a two-sided maximal ideal of $R$ of finite codimension which does not contain $s$.

Proof. Let $J \subset R_{S}$ be a two-sided ideal of finite codimension. Then $I:=$ $J \cap R$ has finite codimension in $R$, hence $I^{2}=I$ by assumption. Moreover, $J=I R_{S}$ and so $J^{2}=\left(I R_{S}\right)\left(I R_{S}\right) \supset I^{2} R_{S}=I R_{S}=J$, proving (1) by Theorem 3. Moreover, $R / I \subset R_{S} / J$ and $R_{S} / J=(R / I)_{\bar{S}}$ where $\bar{S}$ is the image of $S$ in $R / I$. Now Lemma 3 below shows that $R / I \stackrel{\simeq}{\longrightarrow} R_{S} / J$, proving (2).

For (3) we can assume, by induction, that $S=\left\{s^{i} \mid i=0,1,2, \ldots\right\}$. We already know from (1) that every finite dimensional representation of $R_{S}$ is completely reducible. It remains so show that the intersection in $R_{S}$ of the maximal two-sided ideals of finite codimension is zero. Put

$$
\mathcal{M}:=\{M \subset R \mid M \text { a two-sided maximal ideal of finite codim, } s \notin M\} .
$$

For every $M \in \mathcal{M}$ we have $R_{S} M R_{S}=R_{S} M=M R_{S}$. In fact, given $m \in M$ and $i \geq 0$ we have $s^{i} m=m^{\prime} s^{i}$ and $m s^{i}=s^{i} m^{\prime \prime}$ for suitable $m^{\prime}, m^{\prime \prime} \in R$, because $s$ is normalizing. Since the image of $s$ in $R / M$ is non-zero and normalizing, we see that $s$ is invertible modulo $M$ and so $m^{\prime}, m^{\prime \prime} \in M$. This implies that $m s^{-i} \in R_{S} M$ and $s^{-i} m \in M R_{S}$ which proves the claim. Moreover, it follows that $(R / M)_{\bar{S}}=R / M$ where $\bar{S}$ is the image of $S$ in $R / M$, and so $R / M \stackrel{\simeq}{\longrightarrow} R_{S} / R_{S} M R_{S}$.

We now claim that $\bigcap_{M \in \mathcal{M}} R_{S} M=(0)$. Let $a=r s^{-i}$ be an arbitrary element in $\bigcap_{M \in \mathcal{M}} R_{S} M$. Then $r \in R \cap\left(\bigcap_{M \in \mathcal{M}} R_{S} M\right)=\bigcap_{M \in \mathcal{M}} M$. Putting

$$
\mathcal{M}^{\prime}:=\{M \subset R \mid M \text { a two-sided maximal ideal of finite codim, } s \in M\}
$$

we have, by assumption,

$$
\bigcap_{M \in \mathcal{M}} M \cap \bigcap_{M \in \mathcal{M}^{\prime}} M=(0) .
$$

Since $s \in \bigcap_{M \in \mathcal{M}^{\prime}} M$ we see that $r s=0$ for every $r \in \bigcap_{M \in \mathcal{M}} M$. Hence $a=r s^{-i}=(r s) s^{-i-1}=0$. This completes our proof.

Lemma 3. Let $A$ be a left or right Artinian algebra and $S \subset A$ an Ore set. Then the canonical map $A \rightarrow A_{S}$ is surjective.

Proof. This is clear since a non 0-divisor in an Artinian algebra is invertible.

Example 2. The assumptions of part (3) of Proposition 4 are necessary as shown be the following examples. Let $\mathfrak{g}$ be a simple (complex) Lie algebra and $\mathfrak{U}(\mathfrak{g})$ its enveloping algebra. If we choose for $S$ the set of all non-zero 
central elements then $S$ consists of normalizing elements, but $\mathfrak{U}(\mathfrak{g})_{S}$ is a simple ring, hence not FCR.

Putting $S=\left\{s^{i} \mid i=0,1,2 \ldots\right\}$ where $s$ a nilpotent element of $\mathfrak{g}$ then $S$ is an Ore set. (This was first observed by Lepowsky and follows from the fact that $\operatorname{ad}(s)$ is locally nilpotent in $\mathfrak{U}(\mathfrak{g})$.) But $\mathfrak{U}(\mathfrak{g})_{S}$ has no finite dimensional representation and so $\mathfrak{U}(\mathfrak{g})_{S}$ is not FCR.

\section{References}

[Dix64] Dixmier, J., Les $C^{*}$-algèbres et leur représentations (Gauthier-Villars \& Cie., Paris 1964)

[Dix77] Dixmier, J., Enveloping algebras (North-Holland Publishing Company, Amsterdam - New York - Oxford 1977)

[Fa87] Farkas, D., Semisimple representations and affine rings, Proc. Amer. Math. Soc. 101 (1987) 237-238

[KiS00] Kirkman, E., Small, L.W., Examples of FCR-algebras, Preprint (2000)

[KrS94] Kraft, H., Small, L.W., Invariant algebras and completely reducible representations, Math. Research Letters 1 (1994) 297-307

[KSW99] Kraft, H., Small, L.W., Wallach, N.R., Hereditary properties of direct summands of algebras, Math. Research Letters 6 (1999) 371-375

[La91] Lam, T.Y., A First Course in Noncommutative Rings (Springer-Verlag, New York - Berlin - Heidelberg 1991), Graduate Texts in Mathematics vol. 131

[La98] Lam, T.Y., Lectures on Modules and Rings (Springer-Verlag, New York - Berlin - Heidelberg 1998), Graduate Texts in Mathematics vol 189

[MvB98] Musson, I.M., Van den Bergh, M. Invariants under tori of rings of differential operators and related topics (American Mathematical Society, Providence, RI, 1998), Memoirs of the AMS vol. 650

[Sch00] Schwarz, G.W., Finite dimensional representations of invariant differential operators, in preparation (2000)

[SSW85] Small, L.W., Stafford, J.T., Warfield Jr., R.B., Affine algebras of Gelfand-Kirillov dimension one are PI, Math. Proc. Camb. Phil. Soc. 97 (1985) 407-414 